\title{
Toxicidade hepática é rara em pacientes com artrite reumatoide usando terapia combinada de leflunomida e metotrexato
}

\author{
Jorge Augusto Nunes Rodrigues Alves ${ }^{1}$, Sonia Cristina de Magalhães Souza Fialho², \\ Edelton Flávio Morato ${ }^{3}$, Gláucio RicardoWerner de Castro ${ }^{4}$, Adriana Fontes Zimmermann, \\ Giovana Gomes Ribeiro ${ }^{2}$, Fabrício Souza Neves², Ivânio Alves Pereira ${ }^{6}$
}

\begin{abstract}
RESUMO
Objetivo: De acordo com alguns estudos, a associação de leflunomida (LEF) a pacientes portadores de artrite reumatoide não responsivos a metotrexato (MTX) aumentou a eficácia do tratamento, elevando, porém, o risco de toxicidade hepática. Este estudo objetiva avaliar a incidência de toxicidade hepática no tratamento da artrite reumatoide ativa usando terapia combinada de LEF e MTX em comparação com monoterapia com MTX. Métodos: Entre fevereiro e setembro de 2009, foram arrolados 97 pacientes consecutivos acompanhados pelo Hospital Universitário da Universidade Federal de Santa Catarina, Brasil. Pacientes com artrite reumatoide em uso de MTX somente ou em combinação com LEF tiveram seus prontuários sistematicamente revisados. As enzimas alanino/aspartato aminotransferases foram analisadas retrospectivamente desde o tratamento com MTX ou MTX mais LEF. Hepatotoxicidade foi definida como um aumento das enzimas hepáticas acima de duas vezes o limite superior da normalidade. Resultados: 71 pacientes foram incluídos no estudo: 36,6\% usavam 20-25 mg/semana de MTX e 63,4\% usavam 20-25 mg/semana de MTX associado a $20 \mathrm{mg} /$ dia de LEF. Dos pacientes em terapia combinada, $11,1 \%$ tinham níveis anormais das enzimas hepáticas versus $11,5 \%$ daqueles em monoterapia $(\mathrm{P}=1,0)$. Níveis anormais de aminotransferases têm sido observados em pacientes com artrite reumatoide tanto em monoterapia com MTX quanto com LEF. Em nosso estudo, não encontramos diferença entre as percentagens de elevação das aminotransferases em pacientes tratados somente com MTX ou com terapia combinada. Conclusão: A combinação de MTX e LEF em pacientes com artrite reumatoide é geralmente segura e bem tolerada.
\end{abstract}

Palavras-chave: artrite reumatoide, metotrexato, toxicidade de drogas.

[Rev Bras Reumatol 2011;51(2):138-144] CElsevier Editora Ltda.

\section{INTRODUÇÃO}

A artrite reumatoide (AR) é uma doença autoimune comum que se caracteriza por inflamação crônica das articulações sinoviais. Na maioria dos casos, leva à formação de pano, que culmina em destruição articular. Diagnóstico precoce acompanhado por uso agressivo de drogas antirreumáticas que modificam o curso da doença (DARMDs) têm mostrado efeito favorável no curso da doença. ${ }^{1}$ Além disso, combinações de DARMDs têm mostrado maior habilidade para desacelerar a progressão da doença. ${ }^{2,3}$

O metotrexato (MTX) é a DARMD mais eficaz usada na AR, com baixa toxicidade e excelente tolerância. Infelizmente, o MTX isolado com frequência não controla por completo a atividade da doença. Devido à alta taxa de falha da monoterapia na $\mathrm{AR}$ e à complexidade da patogênese da doença, ênfase crescente vem sendo dada às combinações de agentes terapêuticos que inibem diferentes alvos fisiopatológicos da doença. ${ }^{4}$

Recebido em 8/10/2010. Aprovado, após revisão, em 14/1/2011. Os autores declaram a inexistência de conflitos de interesse. Comitê de Ética: FR239691.

Universidade Federal de Santa Catarina - UFSC, Brasil.

1. Médico - Hospital Municipal Antonio Martins da Costa

2. Doutora em Ciências da Saúde pela Universidade de São Paulo - Médica assistente do Núcleo de Reumatologia da UFSC

3. Médico-imunologista - Professor de Imunologia da UFSC

4. Mestre em Ciências da Saúde pela UFSC - Médico-assistente do Núcleo de Reumatologia da UFSC

5. Mestre em Ciências pela UFSC - Médica-assistente do Núcleo de Reumatologia da UFSC

6. Doutor em Ciências pela USP - Chefe do Núcleo de Reumatologia da UFSC

Correspondência para: Sonia Cristina de Magalhães Souza Fialho.

R. Virgílio Várzea, 1510, BI. H , apto101. Florianópolis, SC, Brasil. Zip Code: 88032-001. E-mail: fialhoson@gmail.com. 
Considerando-se seu mecanismo de ação, leflunomida (LEF) é útil em combinação com MTX. Diferentemente da LEF, o MTX nas dosagens usadas para tratamento de AR parece ter pouco efeito na proliferação de células $\mathrm{T}$, mas inibe fortemente a síntese celular de poliaminas e promove liberação de adenosina, efeitos que limitam a inflamação e destruição articular. ${ }^{5}$ Além disso, um recente estudo in vitro sugere que o MTX promove apoptose de células $T$ ativadas, uma ação que complementaria o efeito da LEF para limitar proliferação de célula T. ${ }^{6}$ De acordo com alguns estudos, a adição de LEF ao tratamento de não respondedores ao MTX melhorou a eficácia, mas aumentou o risco de efeitos colaterais gastrointestinais e toxicidade hepática. ${ }^{7}$

O presente estudo foi realizado para avaliar a segurança hepática quanto à adição de LEF ao tratamento com MTX em pacientes com AR ativa que apresentaram resposta inadequada ao MTX isolado.

\section{MATERIAL E MÉTODOS}

Trata-se de estudo transversal realizado entre fevereiro e setembro de 2009 com 97 pacientes consecutivos acompanhados no Hospital da Universidade Federal de Santa Catarina, Brasil. O critério de inclusão consistiu em atender aos critérios de diagnóstico para AR do Colégio Americano de Reumatologia (ACR) ${ }^{8} \mathrm{e}$ estar em uso de MTX isolado ou em combinação com LEF. Foi permitido o tratamento com doses estáveis de anti-inflamatórios não esteroides (AINES) e prednisona em doses inferiores a $15 \mathrm{mg} /$ dia. Todos os pacientes receberam suplementação com ácido fólico, $5 \mathrm{mg}$ uma ou duas vezes por semana.

Os critérios de exclusão foram o uso concomitante de outros DARMDs, agentes biológicos, ou drogas hepatotóxicas, e abuso de álcool. Pacientes com resultados de testes de função hepática persistentemente anormais foram também excluídos. Outras razões de exclusão foram distúrbios hematopoiéticos, infecção pelo vírus da imunodeficiência humana e infecção pelos vírus da hepatite B ou C.

Os prontuários dos pacientes incluídos foram sistematicamente revisados através de um questionário avaliando dados demográficos, variáveis clínicas e uso de medicamentos.

As variáveis laboratoriais testadas foram as enzimas hepáticas alanina aminotransferase e aspartato aminotransferase (ALT/AST, respectivamente) um mês após o início do tratamento com MTX e MTX mais LEF e a cada três meses. Hepatotoxicidade foi definida como um aumento maior do que duas vezes o limite superior da normalidade de AST ou ALT.

No estudo, 97 pacientes com AR foram registrados, mas 26 pacientes foram excluídos devido a várias razões: um tinha hepatite B; cinco suspenderam o MTX muito cedo sem relato de efeitos adversos; três faziam uso de outras combinações de DARMDs; e 17 estavam em tratamento biológico. O tratamento com MTX como monoterapia ou em combinação com LEF foi iniciado apenas nos pacientes com provas de função hepática normais.

Este estudo foi submetido à aprovação institucional, e todos os participantes forneceram consentimento livre informado.

As variáveis numéricas são apresentadas como média e desvio-padrão (DP) e comparadas usando-se o teste $t$ de Student para as diferenças. As variáveis categóricas foram comparadas entre os grupos usando-se o teste do Qui-quadrado ou o teste exato de Fisher. Os resultados foram avaliados de acordo com os valores normais estabelecidos e foram subsequentemente classificados como elevados ou normais. O nível de significância estatística adotado foi $\mathrm{P}<0,05$. Todas as análises estatísticas foram realizadas com o programa EpiInfo 6.04.

\section{RESULTADOS}

Os pacientes foram distribuídos em dois grupos de acordo com o tratamento em uso: MTX ( $\mathrm{n}=26$ ou $36,6 \%$ ) ou MTX + LEF $(n=45$ ou $63,4 \%)$. Todos os pacientes nos dois grupos estavam usando 20 a $25 \mathrm{mg}$ de MTX uma vez por semana. A dose de LEF usada foi de $20 \mathrm{mg}$ por dia.

Os dados demográficos dos dois grupos de AR revelaram semelhança quanto à idade média $(56,4 \pm 14,5$ versus $54,2 \pm 13,6 ; \mathrm{P}=0,51)$ e quanto à frequência do gênero feminino $(88,5 \%$ versus $91,1 \% ; \mathrm{P}=0,71)$. A maioria dos pacientes tomava AINES, mas na mesma proporção em ambos os grupos (Tabela 1).

Três dos 26 (11,5\%) pacientes tratados com MTX e cinco dos $45(11,1 \%)$ pacientes tratados com a combinação apresentaram elevação das aminotransferases como definida em nosso estudo (Tabela 1). Nesses pacientes com níveis anormais de aminotransferases, as doses de MTX foram temporariamente reduzidas.

\section{Tabela 1}

Dados demográficos, uso de AINES e elevação de aminotransferases nos dois grupos

\begin{tabular}{lccc}
\hline & MTX $(\mathrm{n}=26)$ & $\mathrm{MTX}+\mathrm{LEF}(\mathrm{n}=45)$ & $\mathrm{P}$ \\
\hline Idade (anos) & $56,4 \pm 14,5$ & $54,2 \pm 13,6$ & 0,51 \\
Sexo feminino (\%) & 88,5 & 91,1 & 0,72 \\
AINES** $(\%)$ & 84,6 & 86,6 & 0,81 \\
ALT/AST*** $>2 \times(\%)$ & 11,5 & 11,1 & 1,0 \\
\hline
\end{tabular}

*Dados expressos como média e desvio-padrão; ${ }^{* \star}$ AINES: anti-inflamatórios não esteroides; ${ }^{\star \star \star A L T / A S T: ~ a l a n i n a / a s p a r t a t o ~ a m i n o t r a n s f e r a s e s . ~}$ 


\section{DISCUSSÃO}

Nos últimos anos, os pesquisadores mostraram que algumas DARMDs podem aumentar a eficácia de MTX quando usadas em combinação. Devido às diferentes vias intracelulares afetadas pelas duas drogas, a combinação de LEF e MTX tem potencial para sinergia bioquímica. A possibilidade de aumento de benefícios deveria ser comparada às possíveis toxicidades dessa combinação. Níveis anormais de aminotransferases têm sido vistos com a monoterapia tanto de $\mathrm{MTX}^{9-12}$ quanto de $\mathrm{LEF}^{13,14} \mathrm{em}$ pacientes com AR.

Nosso estudo não encontrou diferença entre a percentagem de pacientes com AR que apresentaram elevações de aminotransferases durante o tratamento apenas com MTX ou com o tratamento combinado de MTX e LEF.

Um pequeno estudo aberto relatou que a combinação de MTX e LEF determinou considerável melhora clínica e apenas elevações reversíveis nos níveis de aminotransferases. ${ }^{5}$

Curtis et al. ${ }^{15}$ avaliaram pacientes com AR ou artrite psoriásica iniciando terapia com DARMD. Os autores relataram elevações maiores que duas vezes o limite superior de normalidade em $1 \%-2 \%$ dos pacientes em uso de monoterapia com MTX ou LEF e em 5\% dos pacientes em uso da terapia combinada. Após análise multivariada, a combinação de MTX e LEF foi associada a maior risco de hepatotoxicidade, dependendo da dose de MTX: MTX 10-17,5 mg/semana, OR 2,91 (IC 95\% 1,23-6,90); MTX $\geq 20 \mathrm{mg} / \mathrm{semana}$, OR 3,98 (IC 95\% 1,72-9,24).

Kremer et al. ${ }^{16}$ conduziram um estudo controlado por placebo, multicêntrico, randomizado, duplo-cego de 24 semanas, no qual LEF ou placebo era adicionado à já existente terapia com MTX. As taxas de descontinuação foram semelhantes nos dois grupos de tratamento (23,1\% no grupo LEF e $24,8 \%$ no grupo placebo). Níveis elevados de AST maiores do que 1,2 vez o limite superior da normalidade foi observado em $16,9 \%$ do grupo LEF (22 pacientes) e em 4,5\% do grupo placebo (seis pacientes). Ainda com base na elevação máxima, elevações de ALT e AST maiores do que três vezes o limite superior da normalidade foram mais frequentes em qualquer momento durante as 24 semanas do estudo no grupo LEF do que no grupo placebo. Entretanto, todos os níveis elevados de ALT e AST excedendo 1,2 vez o limite superior da normalidade no grupo LEF normalizaram-se para 1,2 vez o limite superior da normalidade ou menos durante o estudo ou após 24 semanas. Neste estudo, a maioria dos pacientes obteve normalização sem nenhum ajuste na dose de LEF ( $58,5 \%$ dos pacientes com níveis anormais de ALT e 59\% dos pacientes com níveis anormais de AST). Três pacientes no grupo LEF e dois pacientes no grupo placebo tiveram que deixar o estudo antes de 24 semanas porque os níveis de ALT e AST aumentaram além da margem de variação definida pelo protocolo de aceitação em testes repetidos (elevações persistentes dos níveis das aminotransferases para mais de duas vezes o limite superior da normalidade em testes repetidos após ajuste de dose ou confirmação de um valor maior do que cinco vezes o limite superior da normalidade). Houve normalização dos resultados dos testes de função hepática na visita de acompanhamento nos três pacientes tratados com LEF. Nos dois pacientes recebendo placebo, os níveis de ALT e AST ainda estavam elevados na visita de acompanhamento, mas normalizaram-se alguns meses depois.

Katchamart et $a .^{7}$ realizaram uma revisão sistemática de ensaios randomizados comparando MTX isolado e em combinação com outras DARMDs não biológicas para avaliar a eficácia e a toxicidade de drogas em adultos com AR. Em 17 de 19 ensaios (1.624 pacientes: 824 no grupo combinação vs. 800 no grupo monoterapia), a terapia combinada resultou em mais desistências devido a reações adversas do que a monoterapia, mas as diferenças só foram significativas apenas para ciclosporina e azatioprina.

Em conclusão, mais estudos são necessários, mas há evidência atual sugerindo que a combinação MTX + LEF em pacientes com AR é em geral segura e bem tolerada. As enzimas hepáticas devem ser monitoradas, mas toxicidade hepática grave é rara e semelhante àquela de $\mathrm{p}$ acientes usando apenas MTX.

\section{REFERENCES}

\section{REFERÊNCIAS}

1. Combe B, Landewe CL, Lukas C, Bolosiu HD, Breedveld F, Dougados $\mathrm{M}$ et al. EULAR recomendations for the management of early Arthritis report of a task force of the European Standing Committee for Internation Clinical Studies Including Therapeutics. Ann. Rheum. Dis 2007; 66:34-45.

2. Sharp JT, Strand V, Leung H, Hurley F, Loew-Friedrich I. Treatment with leflunomide slows radiographic progression of rheumatoid arthritis: results from three randomized controlled trials of leflunomide in patients with active rheumatoid arthritis. Leflunomide Rheumatoid Arthritis Investigators Group. Arthritis Rheum 2000; 43:495-505.

3. Landewe RB, Boers M, Verhoeven AC, Westhovens R, van de Laar MA, Markusse HM et al. COBRA combination therapy in patients with early rheumatoid arthritis: long-term structural benefits of a brief intervention. Arthritis Rheum 2002;46:347-56.

4. Pereira IA, Cruz BA, Xavier RM, Pinheiro GRC, Titton DC, Giorgi RDN et al. Recomendações nacionais baseadas em evidências científicas e opiniões dos especialistas sobre o uso do metotrexato nas doenças reumáticas, especialmente na artrite reumatoide: resultados da iniciativa 3E do Brasil. Rev Bras Reumatol. 2009; 49:346-61. 
5. Weinblatt ME, Kremer JM, Coblyn JS, Maier AL, Helfgott SM, Morrell M et al. Pharmacokinetics, safety, and efficacy of combination treatment with methotrexate and leflunomida in patients with active rheumatoid arthritis. Arthritis Rheum. 1999; 42:1322-8.

6. Genestier L, Paillot R, Fournel S, Ferraro C, Miossec P, Revillard J. Immunosuppressive properties of methotrexate: apoptosis and clonal deletion of activated peripheral T cells. J Clin Invest 1998; 102:322-8.

7. Katchamart W, Trudeau J, Phumethum V, Bombardier C. Efficacy and toxicity of methotrexate (MTX) monotherapy versus MTX combination therapy with non-biological disease-modifying antirheumatic drugs in rheumatoid arthritis: a systematic review and meta-analysis. Ann Rheum Dis 2009; 68:1105-12.

8. Arnett FC, Edworthy SM, Bloch DA, McShane DJ, Fries JF, Cooper NS et al. The American Rheumatism Association 1987 revised criteria for the classification of rheumatoid arthritis. Arthritis Rheum $1988 ; 31: 315-24$.

9. Kremer JM, Kaye GI, Kaye NW, Ishak KG, Axiotis CA. Light and electron microscopic analysis of sequential liver biopsy samples from rheumatoid arthritis patients receiving long-term methotrexate therapy. Followup over long treatment intervals and correlation with clinical and laboratory variables. Arthritis Rheum 1995; 38:1194-203.

10. Susuki Y, Uehara R, Tajima C, Noguchi A, Ide M, Ichikawa Y, Mizushima Y. Elevation of serum hepatic aminotrsnsferases during treatment of rheumatoid arthritis with low-dose methotrexate. Scand J Rheumatol 1999; 28:273-81.
11. Tilling L, Townsend S, David J. Methotrexate and Hepatic Toxicity in Rheumatoid Arthritis and Psoriatic Arthritis. Clin Drug Invest 2006; 26(2):55-62.

12. Salliot C, Heijde D. Long-term safety of methotrexate monotherapy in patients with rheumatoid arthritis: a systematic literature research. Ann Rheum Dis 2009; 68:1100-04.

13. Smolen JS, Kalden JR, Scott DL, Rozman B, Kvien TK, Larsen A et al. Efficacy and safety of leflunomide compared with placebo and sulphasalazine in active rheumatoid arthritis: a double-blind, randomised, multicentre trial. European Leflunomide Study Group. Lancet 1999; 353:259-66.

14. Cohen S, Cannon GW, Schiff M, Weaver A, Fox R, Olsen N et al. Two-year, blinded, randomized, controlled trial of treatment of active rheumatoid arthritis with leflunomida compared with methotrexate. Utilization of Leflunomide in the Treatment of Rheumatoid Arthritis Trial Investigator Group. Arthritis Rhem 2001; 44(9): 1984-92.

15. Curtis JR, Beukelman T, Onofrei A, Cassell S, Greenberg J, Kavanaugh A et al. Elevated liver enzyme tests among rheumatoid arthritis and psoriatic arthritis patients treated with methotrexate and/ or leflunomida. Ann Rheum Dis 2010; 69(1):43-7.

16. Kremer JM, Genovese MC, Cannon GW, Caldwell RJ, Cush JJ, Furst DE et al. Concomitant Leflunomide Therapy in Patients with Active Rheumatoid Arthritis despite Stable Doses of Methotrexate. Annals of Internal Medicine 2002; 137(9):726-33. 